\title{
Apocynin protects mesangial cells from lipopolysaccharide-induced inflammation by exerting heme oxygenase 1-mediated monocyte chemoattractant protein-1 suppression
}

\author{
NIRMAL PRASAD BHATT ${ }^{1}$, JIN-YOUNG PARK ${ }^{1}$, HEE JAE LEE ${ }^{1}$, SUNG-SOO KIM ${ }^{1}$, \\ YONG-SOO KWON ${ }^{2}$ and WANJOO $\mathrm{CHUN}^{1}$ \\ ${ }^{1}$ Department of Pharmacology, College of Medicine, ${ }^{2}$ College of Pharmacy, \\ Kangwon National University, Chuncheon, Gangwon 200-701, Republic of Korea
}

Received March 21, 2017; Accepted August 1, 2017

DOI: $10.3892 /$ ijmm.2017.3090

\begin{abstract}
Renal failure is observed in the pathological progression of sepsis and septic shock. Renal mesangial cells (RMCs) have been implicated in renal failure as a result of producing mediators, such as monocyte chemoattractant protein-1 (MCP-1) in response to lipopolysaccharide (LPS). Mitogen-activated protein kinases (MAPKs) have been demonstrated to mediate the LPS-induced inflammatory response in RMCs. Although previous studies indicated a promising effect of apocynin in various inflammatory conditions, its antiseptic efficacy in mesangial cells remains to be clearly determined. In the present study, the anti-inflammatory effects of apocynin and its underlying mechanism were examined in LPS-challenged RMCs. Apocynin significantly inhibited nitric oxide (NO) production in LPS-challenged RMCs and the expression levels of inducible NO synthase and cyclooxygenase-2. In addition, the level of LPS-induced MCP-1 expression was significantly attenuated with apocynin. Furthermore, apocynin significantly suppressed the activation of MAPKs, such as extracellular signal-regulated kinases $1 / 2$ and $\mathrm{p} 38$, but not c-Jun N-terminal kinases. Apocynin exhibited significantly increased expression of heme oxygenase-1 (HO-1) induction via nuclear factor (erythroid-derived 2)-like-2 (Nrf-2) phosphorylation. Inhibition of HO-1 with zinc protoporphyrin significantly abolished apocynin-induced suppression of MCP-1, indicating that HO-1 is significant in the suppression of MCP-1. Thus, apocynin exerts antiseptic activity via the suppression of pro-inflammatory signaling pathways and the activation of cytoprotective signaling pathways, such as $\mathrm{HO}-1 / \mathrm{Nrf}-2$ in $\mathrm{RMCs}$, indicating that apocynin may present as a promising
\end{abstract}

Correspondence to: Dr Wanjoo Chun, Department of Pharmacology, College of Medicine, Kangwon National University, Hyoja-2, Chuncheon, Gangwon 200-701, Republic of Korea

E-mail:wchun@kangwon.ac.kr

Key words: apocynin, renal mesangial cells, mitogen-activated protein kinases, monocyte chemoattractant protein-1, heme oxygenase-1 candidate for in vivo evaluation of a therapeutic agent for inflammation-associated renal disorders.

\section{Introduction}

Sepsis and septic shock are associated with systemic inflammatory response, and eventually lead to multiple organ dysfunctions and mortality (1). The most common cause of sepsis is exposure to bacterial structural pathogens resulting in the excessive production of pro-inflammatory mediators, such as nitric oxide (NO) and cyclooxygenase-2 (COX-2), and cytokines, such as tumor necrosis factor- $\alpha$ (TNF- $\alpha$ ) and interleukin-1 $\beta$ (IL-1 $\beta$ ) by aberrant activation of multiple immune cells (1). Renal mesangial cells (RMCs) within the glomerulus are significant in the pathogenesis of various types of renal disease (2). Previous experimental studies have demonstrated that endotoxins activate RMCs (3). Furthermore, RMCs produce sepsis-associated mediators, such as TNF- $\alpha$ and monocyte chemoattractant protein-1 (MCP-1) in response to lipopolysaccharide (LPS) (4). MCP-1, a type of chemokine, contributes to the migration and infiltration of monocytes/macrophages in glomeruli under pathophysiological conditions (5). Renal damage, such as glomerulonephritis, a systemic response to infection, occurs in critically ill sepsis and septic shock patients (6). The increased expression level of MCP-1 was observed during the pathogenesis of renal disease models, such as LPS-induced septic kidney and LPS-challenged macrophage cells $(7,8)$. Recently, aberrant production of pro-inflammatory mediators and cytokines has been demonstrated to be involved in the aggravation of kidney damage under septic conditions (9). Our previous finding indicates that natural compounds possessing anti-inflammatory potential inhibit the expression levels of inducible NO synthase (iNOS) and COX-2, and the secretion of TNF- $\alpha$ and IL-1 $\beta$ in LPS-challenged macrophages $(10,11)$. In addition, it has been reported that mesangial cells protect themselves from the LPS-induced overproduction of COX-2 by enhancing heme oxygenase-1 (HO-1) expression under septic conditions (7). It is important to clearly understand the role of mesangial cells in sepsis given that they perform multiple roles during its progression. Therefore, suppression of these pro-inflammatory mediators and cytokines is useful 
for the treatment of inflammation-associated renal pathogenic conditions.

Nuclear factor (erythroid-derived 2)-like-2 (Nrf-2) is the key transcription factor required for cellular defense against oxidative stress. It has been reported that Nrf-2 exerts cytoprotective effects in septic mice models (12). Caffeic acid phenethyl ester (CAPE) has been reported to contribute to the regulation of peripheral immune functions, expressing HO-1 via the regulation of $\mathrm{Nrf}-2$, a major transcription factor of HO-1 (13). HO-1 is induced by a number of oxidative stresses and exhibits a key cytoprotective role against oxidative cellular injuries. Recent studies have indicated that LPS-challenged macrophages protect themselves from overproduction of inflammatory mediators, such as COX-2 by enhancing HO-1 expression levels (14). In addition, it has been reported that HO-1 inhibits the excessive production of cytokines, such as TNF- $\alpha$ in LPS-challenged RAW264.7 cells (15). Furthermore, HO-1 induction improved animal survival in lethal endotoxemia (12). The evidence indicated that upregulation of HO-1 is associated with significant activation of mitogen-activated protein kinases (MAPKs) and Nrf-2 in animal cells (16).

Apocynin (4-hydroxy-3-methoxyacetophenone), originally isolated from the root extract of Himalayan herb, Picrorhiza kurroa Royle (Scrophulariaceae), inhibits the production of superoxide by generating NADPH oxidase enzyme (17). The aim of the present study was to gain novel insight into the anti-inflammatory properties of apocynin in RMCs given that previous studies demonstrated that apocynin exhibits various pharmacological properties, such as in the pathogenesis of inflammatory skin diseases (18), ulcerative colitis (19), hyperoxaluria-induced nephrolithiasis (20) and acute lung injury (21), and is beneficial for the treatment of osteoarthritis and rheumatoid arthritis (22). The anti-inflammatory mechanisms of apocynin in RMCs are yet to be identified. Therefore, in the present study, the possible protective effect of apocynin and its underlying mechanisms were investigated in LPS-challenged RMCs.

\section{Materials and methods}

Reagents and cell culture. Bacterial LPS from Escherichia coli serotype 055: B5 and apocynin (4'-hydroxy-3'-methoxyacetophenone) were purchased from Sigma-Aldrich (Merck KGaA, Darmstadt, Germany). Apocynin was dissolved in dimethyl sulfoxide and the desired concentration was added to the culture medium of rat mesangial cells, mentioned below. Rat kidney mesangial cells were obtained from the American Type Culture Collection (cat. no. CRL-2573; Manassas, VA, USA) and maintained in HyClone Dulbecco's modified Eagle's medium (GE Healthcare Life Sciences, Logan, UT, USA) supplemented with $15 \%$ fetal bovine serum, $100 \mathrm{U} / \mathrm{ml}$ penicillin, $100 \mathrm{mg} / \mathrm{ml}$ streptomycin (both from Gibco; Thermo Fisher Scientific, Inc., Waltham, MA, USA) and $0.4 \mathrm{mg} / \mathrm{ml}$ G418 disulphate (Duchefa Biochemie, Haarlem, Netherlands) at $37^{\circ} \mathrm{C}$ in $5 \% \mathrm{CO}_{2}$. At $\sim 75 \%$ confluence, mesangial cells were incubated with serum free media and treated with various concentrations of apocynin $(62.5,125,250$ and $500 \mu \mathrm{M})$.

Cell viability assay. The mesangial cells were seeded at $5 \times 10^{4} /$ well in 96 -well plates and incubated at $37^{\circ} \mathrm{C}$ under $5 \%$
$\mathrm{CO}_{2}$ and $95 \%$ humidified air until reaching $>80 \%$ cell confluence. The cells were treated with apocynin for $1 \mathrm{~h}$ prior to the addition of LPS for $24 \mathrm{~h}$. Cell viability was determined using cell counting kit-8 (CCK-8) assay (cat no. CK04; Dojindo Molecular Technologies, Inc, Rockville, MD, USA), which produces a water-soluble formazan dye in living cells. The quantity of formazan dye generated by dehydrogenases in cells is directly proportional to the number of living cells. CCK-8 solution $(10 \mu \mathrm{M})$ was added to each well and the plate was incubated $1-4 \mathrm{~h}$ at $37^{\circ} \mathrm{C}$ incubator. Absorbance was measured at a wavelength of $450 \mathrm{~nm}$ using a microplate reader. The results were expressed as the percentage of surviving cells over control cells.

Nitrite quantification assay. Accurate quantitative measurement of NO production in the biological samples was estimated by measuring the quantity of nitrite, a stable metabolite of NO, using the Griess assays. Subsequent to apocynin-pretreated RMCs being stimulated with LPS in at $5 \times 10^{5} /$ well in 12 -well plates for $24 \mathrm{~h}, 100 \mu \mathrm{l}$ cell supernatant was combined with an equal volume of Griess reagent (Sigma-Aldrich, St. Louis, MO, USA). The absorbance was measured at a wavelength of $550 \mathrm{~nm}$ using a microplate reader and sodium nitrite served as a standard. The results were expressed as the percentage of released NO from LPS-stimulated RMCs, and a standard curve was established.

Western blot analysis. The cells were seeded at $2.5 \times 10^{6} /$ well in $60-\mathrm{mm}$ cell culture plates and pretreated with apocynin for $1 \mathrm{~h}$. Following treatment, the cells were stimulated with LPS for various durations. Then the cells were washed with $1 \mathrm{X}$ phosphate-buffered saline and lysed in PRO-PREP lysis buffer (Intron Biotechnology, Inc., Seongnam, Korea). Samples from the cell lysates were denatured with sonication on ice and equal amounts of protein were separated on $10 \%$ sodium dodecyl sulfate (SDS)-polyacrylamide gel. Proteins were transferred to polyvinylidene fluoride membrane (Merck KGaA) and blocked in 5\% skimmed milk or $3 \%$ bovine serum albumin (BSA) in Tris-buffered saline and Tween-20 (TBST) for $1 \mathrm{~h}$ at room temperature. The membranes were probed with specific antibodies against MCP-1 (1:1,000; cat. no. ab7202; Abcam, Cambridge, MA, USA), iNOS (1:1,000; cat. no. 610333, BD Transduction Laboratories, San Jose, CA, USA), COX-2 (1:1,000; cat. no. 48425; Cell Signaling Technology, Inc., Danvers, MA, USA), phosphorylated-Nrf2 (1:1,000; cat. no. bs-2013R; Bioss, Beijing, China), HO-1 (1:500; cat. no. ab13248; Abcam), Akt (cat. no. 9272), phosphorylated-Akt (cat. no. 9271), extracellular signal-regulated kinase 1/2 (Erk1/2) (cat. no. 4695), phosphorylated-Erk1/2 (cat. no. 9101), p38 (cat. no. 9212), phosphorylated-p38 (cat. no. 9211), phosphorylated-c-Jun N-terminal kinase (cat. no. 9251), JNK (1:1,000; cat. no. 9252) (all from Cell Signaling Technology, Inc.), and $\beta$-actin $(1: 2,500$; cat. no. A5441, Sigma-Aldrich; Merck KGaA) were diluted either in 5\% skimmed milk or 3\% BSA. After thoroughly washing with TBST, the membranes were incubated with horseradish peroxidase (HRP)-conjugated goat anti-rabbit IgG (1:2,500, cat. no. 111-035-144) for polyclonal antibodies, or with HRP-conjugated goat anti-mouse IgG secondary antibodies (1:2,500, cat. no. 715-035-150; Jackson ImmunoResearch 

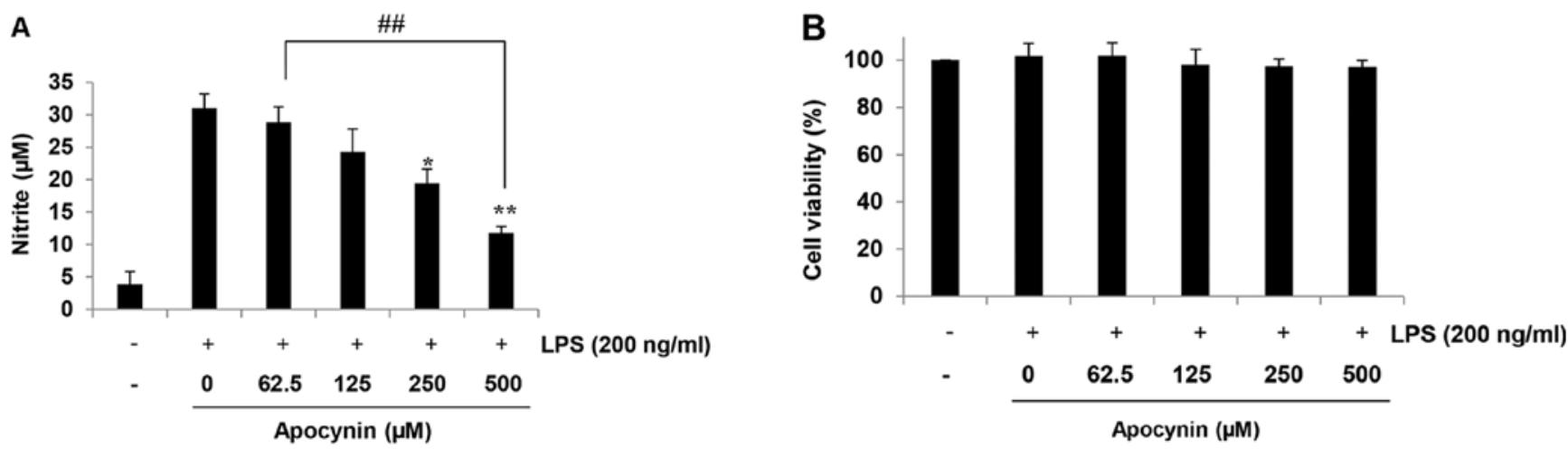

Figure 1. Effects of apocynin on LPS-induced extracellular release of NO in RMCs. (A) Quantitative measurement of NO production was estimated by measuring the quantity of nitrite, a stable metabolite of NO, using Griess assays. Apocynin-treated RMCs were stimulated with LPS in 12 -well plates for $24 \mathrm{~h}$ and $100 \mu \mathrm{l}$ supernatant was mixed with an equal volume of Griess reagent. Apocynin exhibited a significant suppression of LPS-induced NO secretion in a concentration-dependent manner. (B) No significant cell death was observed at the apocynin concentrations used in the present study. Data are expressed as means \pm standard deviation. ${ }^{*} \mathrm{P}<0.05$ and ${ }^{* *} \mathrm{P}<0.01$ vs. LPS alone; ${ }^{\# /} \mathrm{P}<0.01$. LPS, lipopolysaccharide; NO, nitric oxide; RMC, renal mesangial cells.

Laboratories, West Groove, PA, USA). Immunoreactive bands were visualized using an enhanced X-ray film and intensity was measured by ImageQuant 5.2 (GE Healthcare, Waukesha, WI, USA). All values shown in the figures are expressed as the means \pm standard deviation (SD) obtained from at least three independent experiments.

Statistical analysis. The experimental results are expressed as means \pm SD obtained from at least three independent experiments. Statistical significance was analyzed by one-way ANOVA. $\mathrm{P}<0.05$ was considered to indicate a statistically significant difference.

\section{Results}

Apocynin suppresses NO production in LPS-stimulated rat mesangial cells. The anti-inflammatory effects of apocynin were evaluated according to the quantity of NO production in LPS-stimulated RMCs by measuring the quantity of nitrite, a stable metabolite of NO, using Griess reagent. Cells were incubated with the indicated concentrations of apocynin $(62.5,125$, 250 and $500 \mu \mathrm{M}$ ) for $1 \mathrm{~h}$ prior to LPS-treatment. The production of NO in RMCs was increased following LPS incubation for $24 \mathrm{~h}$. However, apocynin significantly inhibited the production of NO in a concentration-dependent manner in LPS-stimulated RMCs (Fig. 1A). Evaluation of the cytotoxicity using CCK-8 assay demonstrated that apocynin-induced cytotoxicity was negligible at concentrations up to $500 \mu \mathrm{M}$ apocynin (Fig. 1B).

Apocynin attenuates LPS-induced iNOS, COX-2 and MCP-1 expression levels in rat mesangial cells. To investigate whether apocynin exhibits inhibitory activity against NO via inhibition of iNOS and COX-2, western blot analysis was performed. LPS treatment resulted in markedly increased expression levels of iNOS and COX-2 in response to LPS. However, pretreatment of apocynin significantly attenuated LPS-induced overproduction of iNOS and COX-2 protein in RMCs (Fig. 2A-C). These findings are consistent with the inhibitory effect of the fractions on NO release (Fig. 1A). As MCP-1 is key in the migration of activated microphages, whether apocynin suppresses the LPS-attenuated expression level of MCP-1 was examined using western blot analysis. To examine the effect of apocynin on LPS-induced MCP-1, mesangial cells were pretreated with apocynin for $\sim 1 \mathrm{~h}$ prior to LPS-treatment for 24 h. LPS-induced MCP-1 expression levels were significantly reduced by apocynin treatment in a concentration-dependent manner (Fig. 2A and D).

Effect of apocynin on LPS-induced phosphorylation of MAPKs in rat mesangial cells. To examine the underlying mechanism by which apocynin exerts its anti-inflammatory effects, the MAPK signaling pathway, which is known to be significantly involved in pro-inflammatory responses, was examined. Cells were pretreated with apocynin at different concentrations for $1 \mathrm{~h}$ and subsequently treated with LPS (200 $\mathrm{ng} / \mathrm{ml}$ ) for $30 \mathrm{~min}$. LPS treatment exhibited a robust activation of all three MAPKs (Fig. 3). Apocynin treatment significantly attenuated the phosphorylation of Erk1/2 and p38 in a concentration-dependent manner (Fig. 3A-C). By contrast, there was no noticeable inhibitory effect of apocynin on phosphorylation of JNK in LPS-induced RMCs (Fig. 3A and D). The suppressive effect of apocynin on the LPS-induced activation of Erk1/2 and p38 signaling pathways strongly indicates that apocynin exerts significant anti-inflammatory effects on mesangial cells. However, further studies are required to clarify the exact mechanism by which apocynin exerts its anti-inflammatory effects via the differential suppression of MAPKs in RMCs.

Apocynin induces phosphorylation of Akt in LPS-stimulated rat mesangial cells. Given that the Akt signaling pathway is significant in cytoprotection against stresses, the effect of apocynin on Akt phosphorylation was examined. Cells were pretreated with apocynin at different concentrations for $1 \mathrm{~h}$ and subsequently treated with LPS (200 ng/ml) for $30 \mathrm{~min}$. LPS treatment demonstrated an increased phosphorylation of Akt, which is considered to be a compensatory response to cellular damage (Fig. 4). Apocynin treatment significantly suppressed LPS-induced phosphorylation of Akt in a concentration-dependent manner (Fig. 4). However, apocynin, although significant, could not reverse the LPS-induced Akt phosphorylation back to the basal level. Further studies are 
A
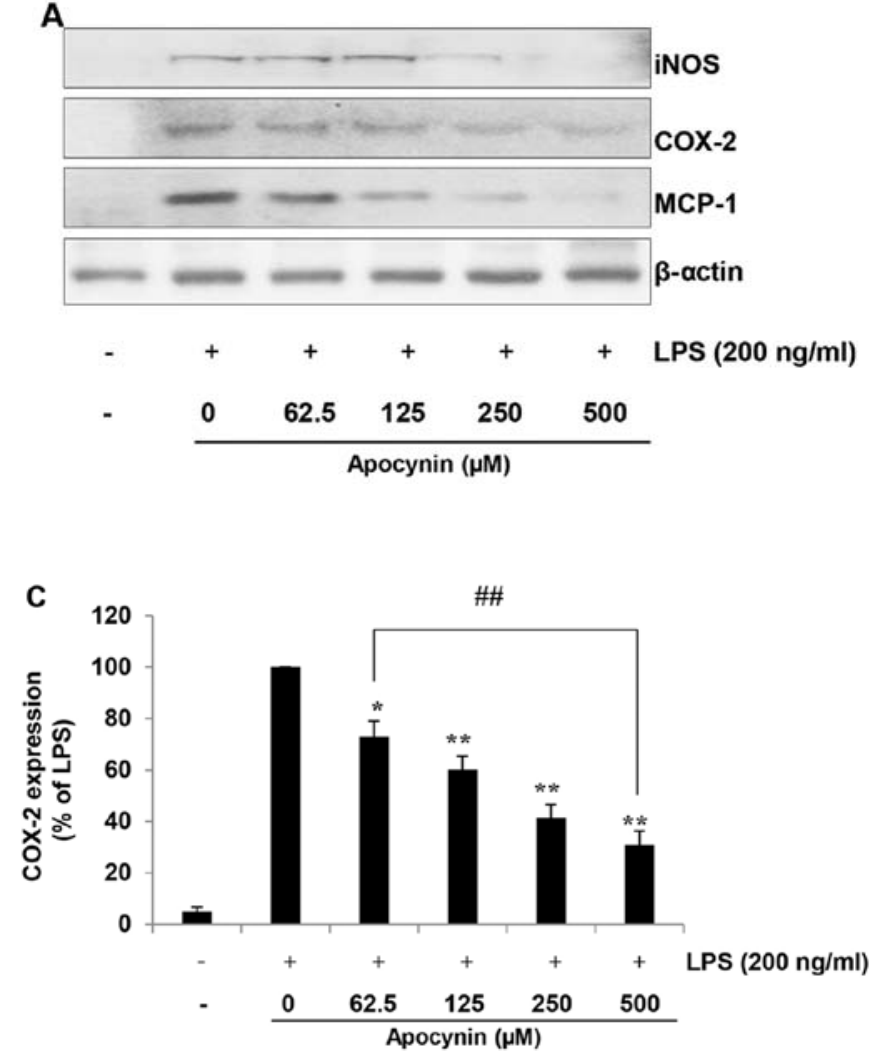
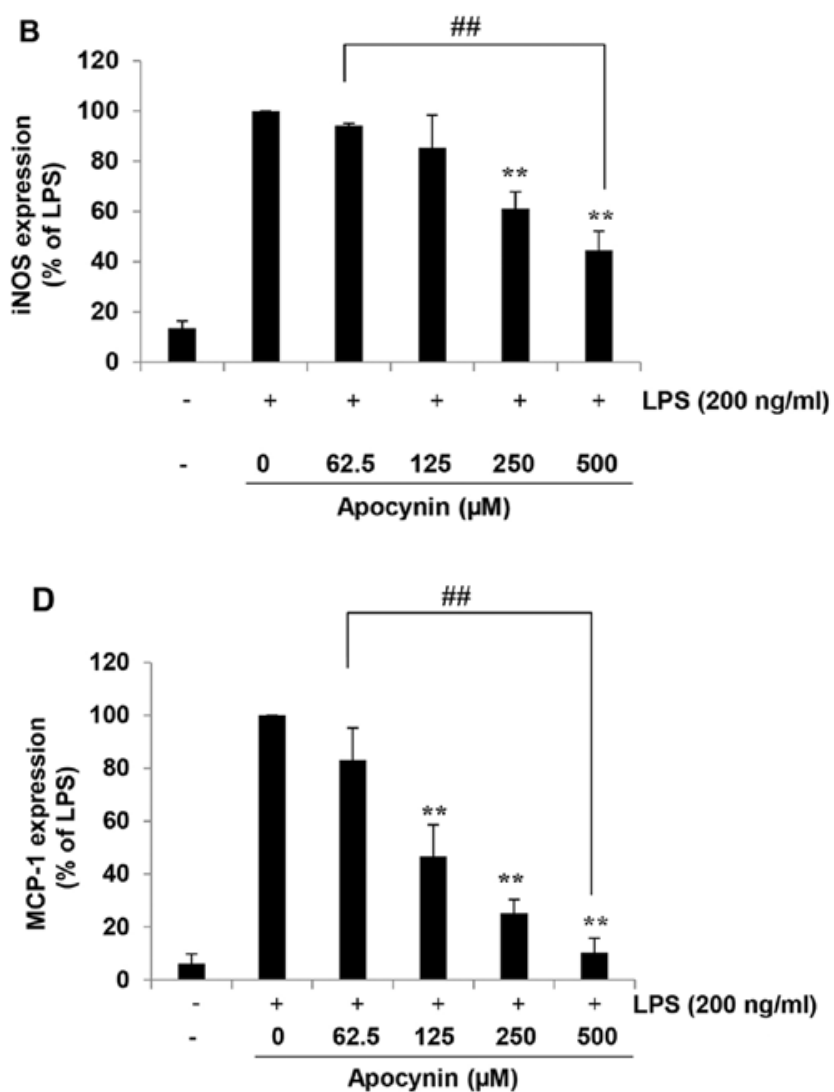

Figure 2. Apocynin inhibits LPS-induced expression levels of iNOS, COX-2 and MCP-1 proteins in RMCs. (A) Cell lysates were subjected to 10\% SDS-PAGE, and the protein expression levels of iNOS, COX-2 and MCP-1 were determined by western blot analysis. The expression levels of iNOS, COX-2 and MCP-1 were markedly increased in response to LPS, and apocynin significantly inhibited the LPS-induced expression of these proteins in a concentration-dependent manner. Quantitative analyses of (B) iNOS, (C) COX-2 and (D) MCP-1 immunoblots. Apocynin significantly suppressed the LPS-induced expression of these proteins. Immunoblots of each protein were obtained from three independent experiments. Data are expressed as means \pm standard deviation. ${ }^{*} \mathrm{P}<0.05$ and ${ }^{* *} \mathrm{P}<0.01$ vs. LPS alone; ${ }^{\# \#} \mathrm{P}<0.01$. LPS, lipopolysaccharide; iNOS, inducible nitric oxide synthase; COX-2, cyclooxygenase-2; MCP-1, monocyte chemoattractant protein-1; RMC, renal mesangial cells.

required to clearly elucidate the exact role of Akt activation in LPS-challenged RMCs.

Apocynin induces $\mathrm{HO}-1$ protein expression via Nrf2 activation in rat mesangial cells. As HO-1 modulates inflammation and inhibits LPS-induced production of pro-inflammatory mediators, and as Nrf2, a transcription factor for HO-1, mediates major cytoprotective responses, the role of apocynin on the HO-1/Nrf2 signaling pathway was examined. RMCs were pretreated with apocynin at different concentrations for $24 \mathrm{~h}$. The level of HO-1 expression was significantly increased in apocynin-treated RMCs in a concentration-dependent manner (Fig. 5A and B). Furthermore, apocynin exhibited increased Nrf2 phosphorylation in accordance with HO-1 expression (Fig. 5C and D). The data strongly indicate that apocynin exerts its anti-inflammatory activity via activation of a cellular cytoprotective pathway.

Effects of HO-1 in LPS-induced MCP-1 expression in rat mesangial cells. To verify the cytoprotective role of apocynininduced upregulation of HO-1 on the LPS-challenged RMCs, the level of MCP-1 expression was examined in the absence and presence of HO-1 activity. RMCs were pretreated with zinc protoporphyrin (Znpp), a HO-1 inhibitor, and subsequently treated with apocynin. Following 24-h incubation with LPS, the expression level of MCP-1 was determined. In the presence of HO-1 activity, apocynin significantly attenuated LPS-induced MCP-1 expression levels in a concentration-dependent manner (Fig. 6). However, apocynin-induced suppression of MCP-1 expression was significantly abolished in the absence of HO-1 activity with Znpp. The data strongly indicates that HO-1 mediates the apocynin-induced suppression of MCP-1 in LPS-challenged RMCs.

\section{Discussion}

In the present study, apocynin was demonstrated to significantly attenuate LPS-induced inflammatory responses via the suppression of MAPK signaling pathways, such as Erk and p38, and the activation of cytoprotective pathways, such as HO-1/Nrf2 in LPS-challenged RMCs. Apocynin suppressed LPS-induced expression of pro-inflammatory mediators, such as iNOS and COX-2. In addition, apocynin significantly attenuated LPS-induced expression of MCP-1, which was abolished with the suppression of $\mathrm{HO}-1$, indicating that $\mathrm{HO}-1$ mediates the suppressive effect of apocynin on MCP-1 expression in LPS-challenged RMCs.

RMCs have been reported to be involved in the autoimmune glomerulonephritis (2), generation of oxygen radicals (23), and glomerular coagulation and fibrinolysis (24). A previous 

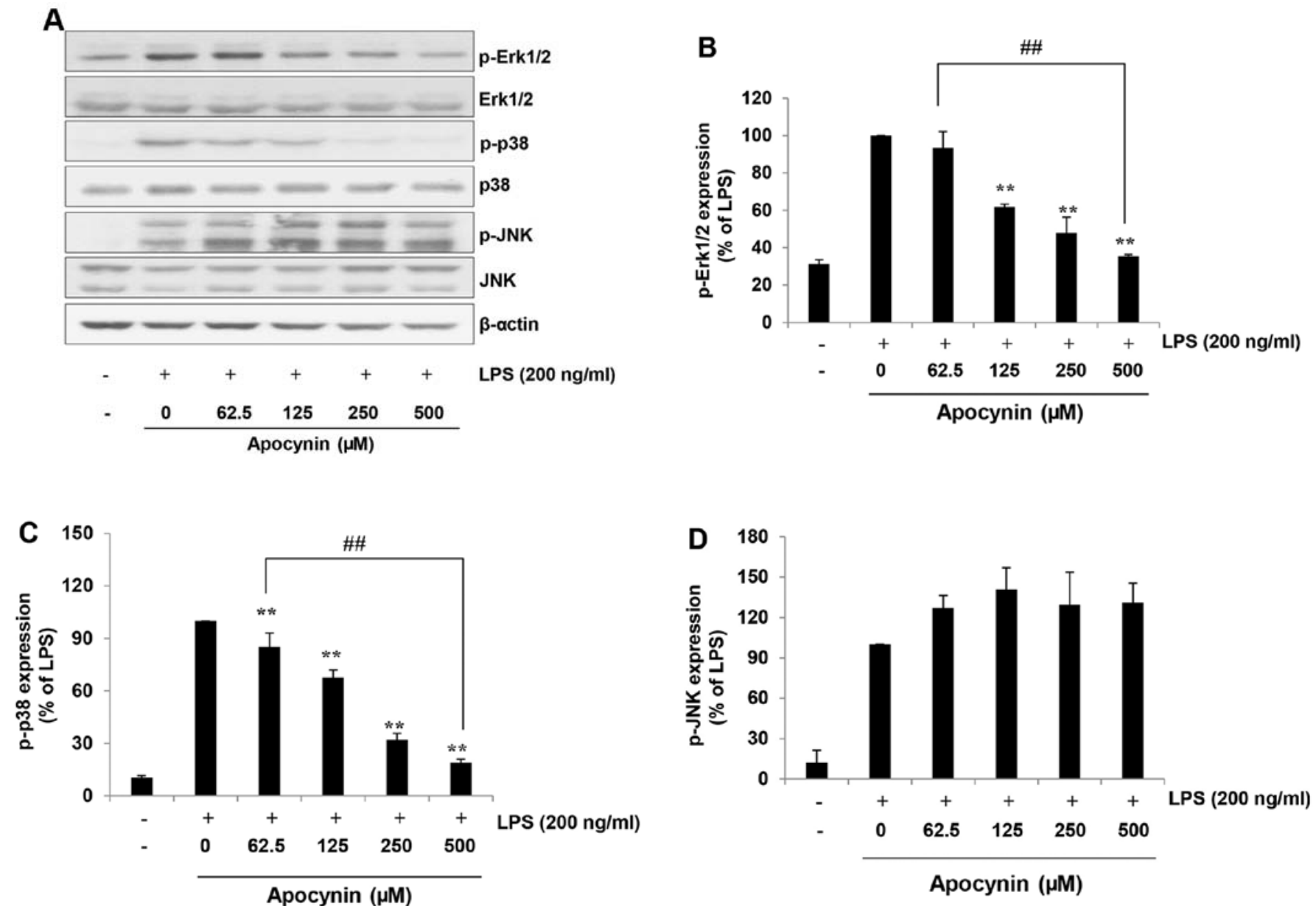

Figure 3. Apocynin attenuates LPS-induced phosphorylation of Erk and p38 in RMCs. The effect of apocynin on LPS-stimulated activation of mitogen-activated protein kinases was measured in RMCs. (A) Representative immunoblots, and quantitative analyses of (B) Erk1/2, (C) p38 and (D) JNK immunoblots Cells were pretreated with apocynin at different concentrations for $1 \mathrm{~h}$ and subsequently treated with LPS for 30 min. Apocynin significantly suppressed the phosphorylation of Erk1/2 and p38 in a concentration-dependent manner. However, there was no significant difference observed on phosphorylation of JNK. Immunoblots of each protein were obtained from three independent experiments. Data are expressed as means \pm standard deviation. ${ }^{* *} \mathrm{P}<0.01 \mathrm{vs}$. LPS alone; ${ }^{\# \#} \mathrm{P}<0.01$. LPS, lipopolysaccharide; Erk1/2, extracellular signal-regulated kinase 1/2; RMC, renal mesangial cells; JNK, c-Jun N-terminal kinase.

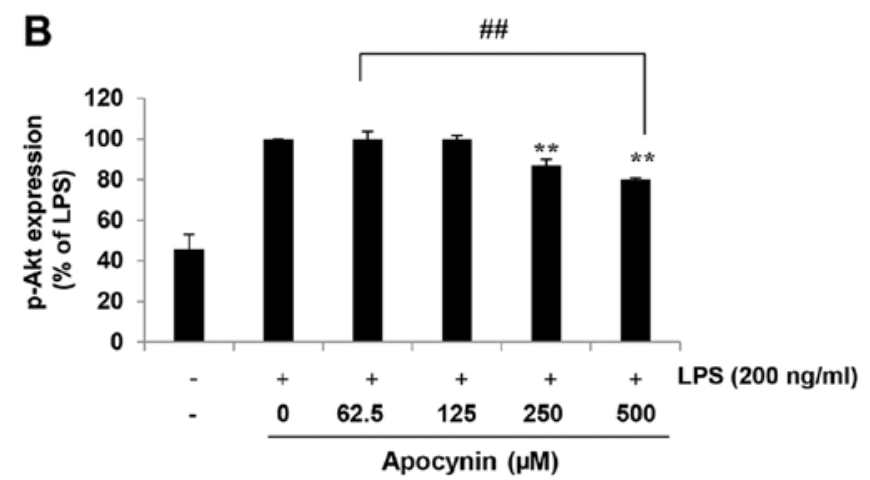

Figure 4. Apocynin attenuates Akt phosphorylation in LPS-induced RMCs. (A) Representative and (B) quantitative analyses of immunoblots. Cells were pretreated with apocynin at different concentrations for $1 \mathrm{~h}$ and subsequently treated with LPS for $30 \mathrm{~min}$. The relative protein expression levels of p-Akt were measured by scanning densitometry and normalized to total Akt. The data indicated that LPS-induced Akt phosphorylation was attenuated by apocynin in a concentration-dependent manner. Immunoblots of each protein were obtained from three independent experiments. Data were expressed as means \pm standard deviation. ${ }^{* *} \mathrm{P}<0.01$ vs. LPS alone; ${ }^{\# \#} \mathrm{P}<0.01$. LPS, lipopolysaccharide; RMC, renal mesangial cells; p, phosphorylated.

study indicated that the suppression of COX-2, iNOS and NO contributes to the anti-inflammatory responses in LPS-treated human renal mesangial cells (25). Consistently, the present study demonstrated that apocynin exerted an inhibitory effect on LPS-induced iNOS and COX-2 expression, and NO production in a concentration-dependent manner.

MCP-1-induced migration and infiltration of macrophages contributes to the aggravation of sepsis and septic shock. MCP-1, 

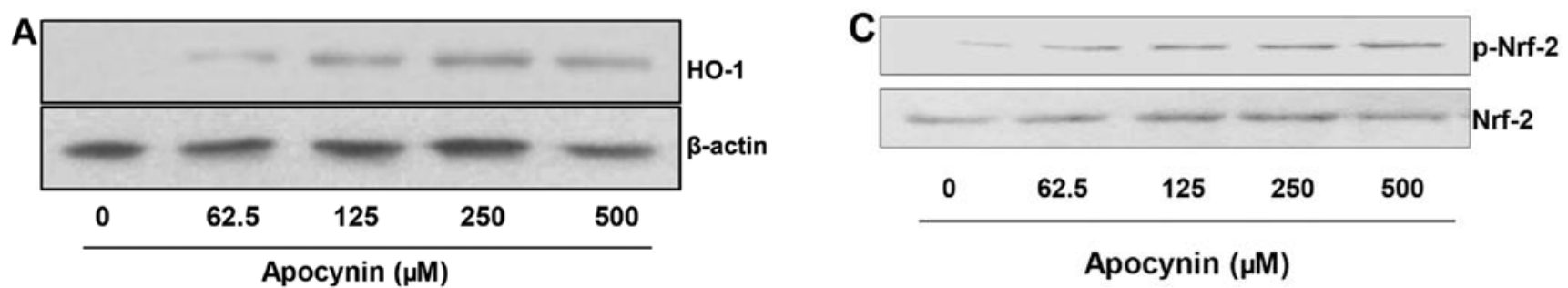

Apocynin ( $\mu \mathrm{M})$
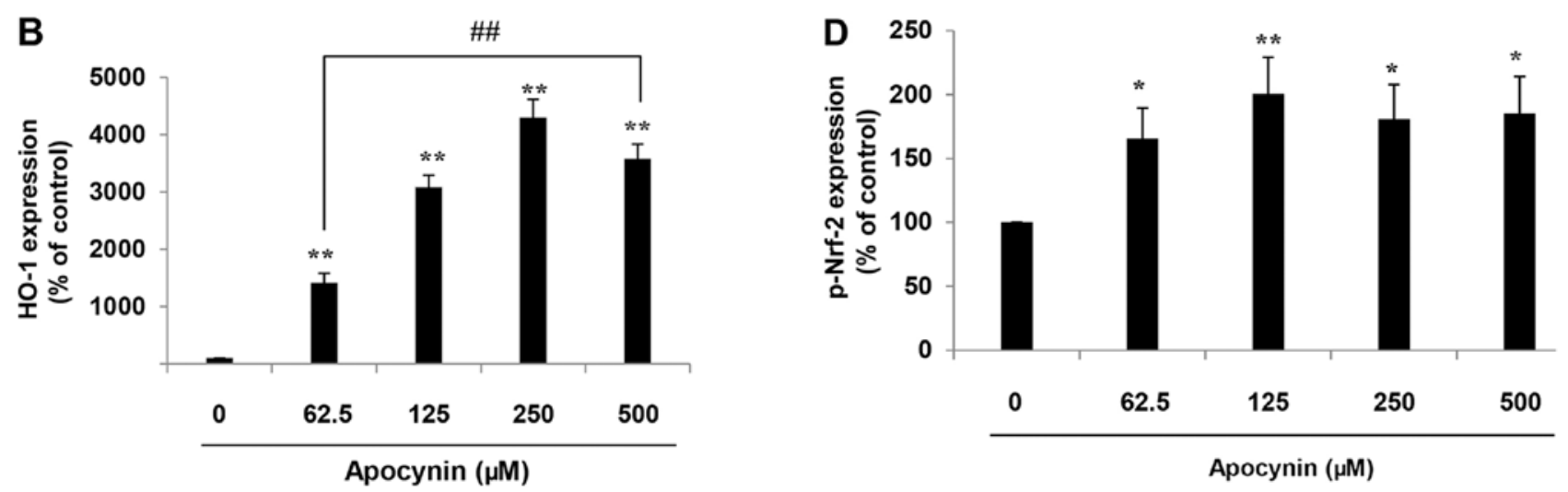

Figure 5. Apocynin induces HO-1 expression via Nrf-2 activation in renal mesangial cells (RMCs). The effect of apocynin on the expression level of (A and B) HO-1 and (C and D) the phosphorylation of Nrf-2 were examined. RMCs were incubated with apocynin for 24 h. A significant increase in HO-1 expression level was observed following apocynin treatment. In addition, apocynin treatment resulted in the significantly increased phosphorylation of Nrf-2, a transcription factor of $\mathrm{HO}-1$. Data were obtained from three independent experiments and expressed as means \pm standard deviation. ${ }^{*} \mathrm{P}<0.05$ and ${ }^{* *} \mathrm{P}<0.01 \mathrm{vs}$. control alone; ${ }^{\# \#} \mathrm{P}<0.01$. HO-1, heme oxygenase-1; Nrf-2, nuclear factor (erythroid-derived 2)-like-2; RMC, renal mesangial cells.

A

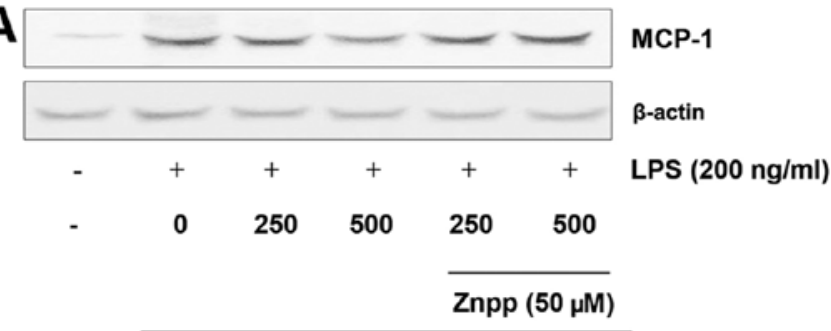

Apocynin ( $\mu \mathrm{M})$

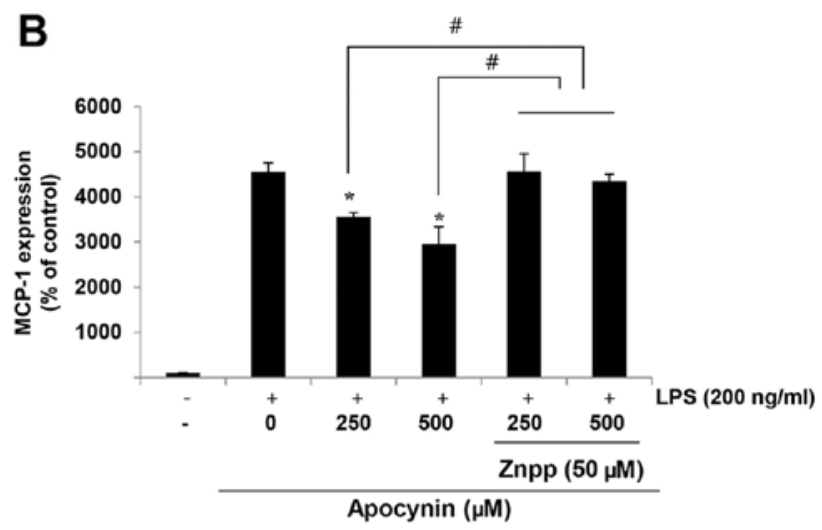

Figure 6. HO-1 mediates the suppression of LPS-induced MCP-1 expression in renal mesangial cells (RMCs). The effect of HO-1 on the expression level of MCP-1 was examined. RMCs were incubated in the absence or presence of Znpp, a HO-1 inhibitor, for 30 min and subsequently treated with apocynin for $1 \mathrm{~h}$ before incubation with LPS ( $200 \mathrm{ng} / \mathrm{ml})$ for $24 \mathrm{~h}$. Znpp abolished apocynin-induced suppression of MCP-1 expression, strongly indicating that apocynininduced HO-1 is significant in MCP-1 suppression. Data were obtained from three independent experiments and expressed as means \pm standard deviation. ${ }^{*} \mathrm{P}<0.05$ vs. LPS alone; ${ }^{\#} \mathrm{P}<0.05$. HO-1, heme oxygenase-1; LPS, lipopolysaccharide; MCP-1, monocyte chemoattractant protein-1; RMC, renal mesangial cells; Znpp, zinc protoporphyrin.

produced by mesangial cells, has been reported to contribute to the pathogenesis of glomerulonephritis (26). The involvement of MCP-1 is not limited to glomerular inflammation, but may be observed in other inflammatory diseases. Various studies have demonstrated that MCP-1 is elevated and has a detrimental role in the development of atherosclerosis (27), inflammatory bowel diseases (28), rheumatoid arthritis (29) and allergic encephalitis (30). Recently, it was reported that trihydroxycinnamic acid suppressed LPS-induced MCP-1 protein expression, resulting in the significant attenuation of LPS-induced septic renal damage (9). In the present study, apocynin significantly inhibited LPS-induced MCP-1 expression levels in RMCs.
The present study demonstrated that among MAPKs, the phosphorylation of Erk1/2 and p38, but not JNK, was attenuated by apocynin with concurrent suppression of inflammatory responses in LPS-challenged RMCs. Previously, a similar study has demonstrated that activation of p38, Erk1/2 and Akt, in response to hydrogen peroxide, was suppressed by apocynin treatment (31). Another consistent finding revealed that only Erk and p38 signaling pathways were involved in the induction of HO-1 (7). The Erk1/2 signaling pathway has been implicated in the development of inflammatory cellular responses in kidney injury (8), as well as in macrophage cells (32). The present study also indicated that Akt phosphorylation was 
attenuated by apocynin treatment in LPS-challenged RMCs. In the present study, activation of the Akt signaling pathway with LPS treatment was considered a compensatory response to cellular damage. Apocynin attenuated the LPS-induced Akt phosphorylation. However, further studies are required to clearly elucidate the exact role of differential suppression on MAPK and Akt signaling pathways in LPS-challenged RMCs.

Nrf-2 has been demonstrated to be key in maintaining cellular homeostasis and regulating inflammatory conditions via cytoprotective enzymes and stress-responsive proteins $(33,34)$. Sulforaphane-treated mice demonstrated Nrf-2 activation and increased HO-1 expression (35), and loss of Nrf-2 signaling increased the susceptibility to inflammation (36). Furthermore, induction of HO-1 resulted in the reduction of oxidative stress by removing free heme, as well as an increase in the level of anti-inflammatory substances (37). In the present study, apocynin, resulting from upregulation of the Nrf-2 signaling pathway, exhibited increased expression levels of HO-1 in RMCs. It has been reported that activation of the HO-1/Nrf-2 signaling pathway may be achieved via the various signaling pathways $(10,12,38)$. Considering the cytoprotective role of HO-1, the induction of HO-1 expression by pharmacological modulation may be a valuable therapeutic target against the progression of sepsis and inflammatory disorders. The present study demonstrated that apocynin resulted in an increase of HO-1 expression levels with subsequent attenuation of inflammation in RMCs. In addition, inhibition of HO-1 with an inhibitor, Znpp, significantly abolished apocynin-induced MCP-1 suppression in RMCs, strongly indicating that HO-1 is critically involved in the suppression of MCP-1 expression levels in RMCs. The data was consistent with previous studies demonstrating that $\mathrm{HO}-1$ inducers were reported to reduce $\mathrm{MCP}-1$ expression levels in sepsis and inflammatory disorders $(39,40)$.

Apocynin, isolated from the root extract of Picrorhiza kurroa, has been reported to perform a range of biological activities, including radical scavenger (41) and antioxidant in vascular cells (31), via the suppression of NADPH oxidase, which is responsible for the generation of endogenous reactive oxygen species (ROS) (42). Apocynin has been reported to inhibit the formation of functional NADPH oxidase complex (41). Aberrant production of NADPH-induced ROS, such as superoxide anion and hydrogen peroxide, mediates injury in various types of inflammatory disorder (41). Furthermore, apocynin was demonstrated to provide blood pressure-lowering effects in hypertensive animals $(43,44)$, as well as exerting renal protective action in rats (45). However, the pharmacological properties of apocynin on RMCs have not been extensively elucidated in LPS-challenged RMCs. The present study is the first, to the best of our knowledge, to demonstrate that apocynin is an effective inducer of HO-1 expression in RMCs. Recently, it was reported that apocynin prevents quinolinic acid-induced neurological damage by increasing glutathione synthesis and Nrf2 mRNA expression levels (46) and that apocynin attenuates diabetes-induced adverse remodeling of left ventricular myocardium (47), indicating the diverse therapeutic properties of apocynin.

In conclusion, the present study clearly demonstrates that apocynin may attenuate kidney dysfunction by suppressing the MCP-1-induced renal infiltration of macrophages via upregulation of the HO-1/Nrf2 signaling pathway in RMCs. In addition, apocynin significantly suppresses LPS-induced overproduction of pro-inflammatory mediators via the inhibition of aberrantly activated MAPK and Akt signaling pathways. These results demonstrate that apocynin may present as a promising candidate for in vivo evaluation of a therapeutic agent for inflammation-associated renal disorders.

\section{Acknowledgements}

The present study was supported by the 2016 Research Grant from Kangwon National University (grant no. 520160299).

\section{References}

1. Singer M, Deutschman CS, Seymour CW, Shankar-Hari M, Annane D, Bauer M, Bellomo R, Bernard GR, Chiche JD, Coopersmith CM, et al: The third international consensus definitions for sepsis and septic shock (sepsis-3). JAMA 315: 801-810, 2016.

2. Radeke $\mathrm{HH}$ and Resch $\mathrm{K}$ : The inflammatory function of renal glomerular mesangial cells and their interaction with the cellular immune system. Clin Investig 70: 825-842, 1992.

3. Almeida WS, Maciel TT, Di Marco GS, Casarini DE, Campos AH and Schor N: Escherichia coli lipopolysaccharide inhibits renin activity in human mesangial cells. Kidney Int 69: 974-980, 2006.

4. Hsing $\mathrm{CH}$, Chou W, Wang JJ, Chen HW and Yeh CH: Propofol increases bone morphogenetic protein-7 and decreases oxidative stress in sepsis-induced acute kidney injury. Nephrol Dial Transplant 26: 1162-1172, 2011.

5. Deshmane SL, Kremlev S, Amini S and Sawaya BE: Monocyte chemoattractant protein-1 (MCP-1): An overview. J Interferon Cytokine Res 29: 313-326, 2009.

6. Poukkanen M, Vaara ST, Pettilä V, Kaukonen KM, Korhonen AM, Hovilehto S, Inkinen O, Laru-Sompa R, Kaminski T, Reinikainen M, et al; FINNAKI study group: Acute kidney injury in patients with severe sepsis in Finnish Intensive Care Units. Acta Anaesthesiol Scand 57: 863-872, 2013.

7. Chen YC, Chen CH, Ko WS, Cheng CY, Sue YM and Chen TH: Dipyridamole inhibits lipopolysaccharide-induced cyclooxygenase- 2 and monocyte chemoattractant protein-1 via heme oxygenase-1-mediated reactive oxygen species reduction in rat mesangial cells. Eur J Pharmacol 650: 445-450, 2011.

8. Nagai T, Urushihara M, Kinoshita Y, Jamba A, Kondo S and Kagami S: Differential regulation of angiotensin II-induced extracellular signal regulated kinase- $1 / 2$ and -5 in progressive glomerulonephritis. Nephrology (Carlton) 21: 950-958, 2016.

9. Lee JW, Kwon JH, Lim MS, Lee HJ, Kim SS, Lim SY and Chun W: 3,4,5-Trihydroxycinnamic acid increases heme-oxygenase-1 (HO-1) and decreases macrophage infiltration in LPS-induced septic kidney. Mol Cell Biochem 397: 109-116, 2014.

10. Lee JW, Bae CJ, Choi Y-J, Kim SI, Kim NH, Lee HJ, Kim SS, Kwon YS and Chun W: 3, 4, 5-Trihydroxycinnamic acid inhibits LPS-induced iNOS expression by suppressing NF- $\kappa \mathrm{B}$ activation in BV2 microglial cells. Korean J Physiol Pharmacol 16: 107-112, 2012.

11. Vo VA, Lee JW, Park JH, Kwon JH, Lee HJ, Kim SS, Kwon YS and Chun W: N-(p-Coumaryol)-tryptamine suppresses the activation of JNK/c-Jun signaling pathway in LPS-challenged RAW264. 7 cells. Biomol Ther (Seoul) 22: 200-206, 2014.

12. Ha YM, Ham SA, Kim YM, Lee YS, Kim HJ, Seo HG, Lee JH, Park MK and Chang KC: $\beta 1$-adrenergic receptor-mediated HO-1 induction, via PI3K and p38 MAPK, by isoproterenol in RAW 264.7 cells leads to inhibition of HMGB1 release in LPS-activated RAW 264.7 cells and increases in survival rate of CLP-induced septic mice. Biochem Pharmacol 82: 769-777, 2011.

13. Fidan H, Sahin O, Yavuz Y, Kilbas A, Cetinkaya Z, Ela Y, Ozen OA and Altuntas I: Caffeic acid phenethyl ester reduces mortality and sepsis-induced lung injury in rats. Crit Care Med 35: 2822-2829, 2007.

14. Park SY, Seetharaman R, Ko MJ, Kim DY, Kim TH, Yoon MK, Kwak JH, Lee SJ, Bae YS and Choi YW: Ethyl linoleate from garlic attenuates lipopolysaccharide-induced pro-inflammatory cytokine production by inducing heme oxygenase-1 in RAW264.7 cells. Int Immunopharmacol 19: 253-261, 2014. 
15. Tsoyi K, Lee TY, Lee YS, Kim HJ, Seo HG, Lee JH and Chang KC: Heme-oxygenase-1 induction and carbon monoxidereleasing molecule inhibit lipopolysaccharide (LPS)-induced high-mobility group box 1 release in vitro and improve surviva of mice in LPS- and cecal ligation and puncture-induced sepsis model in vivo. Mol Pharmacol 76: 173-182, 2009.

16. Park EJ, Lim JH, Nam SI, Park JW and Kwon TK: Rottlerin induces heme oxygenase-1 (HO-1) up-regulation through reactive oxygen species (ROS) dependent and PKC delta-independent pathway in human colon cancer HT29 cells. Biochimie 92 $110-115,2010$

17. Paracatu LC, Zeraik ML, Bertozo LC, Bartolomeu AA, Filho LC, Fonseca LM and Ximenes VF: Synthesis, antioxidant and anti-inflammatory properties of an apocynin-derived dihydrocoumarin. Med Chem 13: 93-100, 2017.

18. Nam YJ, Kim A, Sohn DS and Lee CS: Apocynin inhibits Toll-like receptor-4-mediated activation of NF- $\mathrm{kB}$ by suppressing the Akt and mTOR pathways. Naunyn Schmiedebergs Arch Pharmacol 389: 1267-1277, 2016.

19. Hwang YJ, Lee SJ, Park JY, Chun W, Nam SJ, Park JM, Park SC, Choi DH and Kang CD: Apocynin suppresses lipopolysaccharide-induced inflammatory responses through the inhibition of MAP kinase signaling pathway in RAW264.7 cells. Drug Dev Res 77: 271-277, 2016

20. Sharma M, Kaur T and Singla SK: Role of mitochondria and NADPH oxidase derived reactive oxygen species in hyperoxaluria induced nephrolithiasis: Therapeutic intervention with combinatorial therapy of $\mathrm{N}$-acetyl cysteine and Apocynin. Mitochondrion 27: 15-24, 2016.

21. Abdelmageed ME, El-Awady MS and Suddek GM: Apocynin ameliorates endotoxin-induced acute lung injury in rats. Int Immunopharmacol 30: 163-170, 2016.

22. Lafeber FP, Beukelman CJ, van den Worm E, van Roy JL, Vianen ME, van Roon JA, van Dijk H and Bijlsma JW: Apocynin, a plant-derived, cartilage-saving drug, might be useful in the treatment of rheumatoid arthritis. Rheumatology (Oxford) 38: 1088-1093, 1999.

23. Radeke HH, Meier B, Topley N, Flöge J, Habermehl GG and Resch K: Interleukin 1- $\alpha$ and tumor necrosis factor- $\alpha$ induce oxygen radical production in mesangial cells. Kidney Int 37 767-775, 1990

24. Migliorini A, Ebid R, Scherbaum CR and Anders HJ: The danger control concept in kidney disease: Mesangial cells. J Nephrol 26 : 437-449, 2013

25. Wu F, Zhang W, Li L, Zheng F, Shao X, Zhou J and Li H: Inhibitory effects of honokiol on lipopolysaccharide-induced cellular responses and signaling events in human renal mesangia cells. Eur J Pharmacol 654: 117-121, 2011.

26. Brown Z, Strieter RM, Neild GH, Thompson RC, Kunkel SL and Westwick J: IL-1 receptor antagonist inhibits monocyte chemotactic peptide 1 generation by human mesangial cells. Kidney Int 42: 95-101, 1992

27. Namiki M, Kawashima S, Yamashita T, Ozaki M, Hirase T, Ishida T, Inoue N, Hirata K, Matsukawa A, Morishita R, et al Local overexpression of monocyte chemoattractant protein-1 at vessel wall induces infiltration of macrophages and formation of atherosclerotic lesion: Synergism with hypercholesterolemia. Arterioscler Thromb Vasc Biol 22: 115-120, 2002.

28. Spoettl T, Hausmann M, Herlyn M, Gunckel M, Dirmeier A, Falk W, Herfarth H, Schoelmerich J and Rogler G: Monocyte chemoattractant protein-1 (MCP-1) inhibits the intestinal-like differentiation of monocytes. Clin Exp Immunol 145: 190-199, 2006.

29. Rantapää-Dahlqvist S, Boman K, Tarkowski A and Hallmans G: Up regulation of monocyte chemoattractant protein-1 expression in anti-citrulline antibody and immunoglobulin $\mathrm{M}$ rheumatoid factor positive subjects precedes onset of inflammatory response and development of overt rheumatoid arthritis. Ann Rheum Dis 66: 121-123, 2007.

30. Izikson L, Klein RS, Charo IF, Weiner HL and Luster AD: Resistance to experimental autoimmune encephalomyelitis in mice lacking the CC chemokine receptor (CCR)2. J Exp Med 192: 1075-1080, 2000.

31. Heumüller S1, Wind S, Barbosa-Sicard E, Schmidt HH, Busse R, Schröder K and Brandes RP: Apocynin is not an inhibitor of vascular NADPH oxidases but an antioxidant. Hypertension 51: 211-217, 2008.
32. Vo VA, Lee JW, Chang JE, Kim JY, Kim NH, Lee HJ, Kim SS, Chun W and Kwon YS: Avicularin inhibits lipopolysaccharide-induced inflammatory response by suppressing ERK phosphorylation in RAW 264.7 macrophages. Biomol Ther (Seoul) 20: 532-537, 2012.

33. Lee I-S, Lim J, Gal J, Kang JC, Kim HJ, Kang BY and Choi HJ: Anti-inflammatory activity of xanthohumol involves heme oxygenase-1 induction via NRF2-ARE signaling in microglial BV2 cells. Neurochem Int 58: 153-160, 2011.

34. Surh YJ, Kundu JK and Na HK: Nrf2 as a master redox switch in turning on the cellular signaling involved in the induction of cytoprotective genes by some chemopreventive phytochemicals. Planta Med 74: 1526-1539, 2008.

35. Lin W, Wu RT, Wu T, Khor TO, Wang H and Kong AN: Sulforaphane suppressed LPS-induced inflammation in mouse peritoneal macrophages through Nrf2 dependent pathway. Biochem Pharmacol 76: 967-973, 2008.

36. Yates MS, Tran QT, Dolan PM, Osburn WO, Shin S, McCulloch CC, Silkworth JB, Taguchi K, Yamamoto M, Williams CR, et al: Genetic versus chemoprotective activation of Nrf2 signaling: Overlapping yet distinct gene expression profiles between Keapl knockout and triterpenoid-treated mice. Carcinogenesis 30: 1024-1031, 2009.

37. Kawakami T, Takahashi T, Shimizu H, Nakahira K, Takeuchi M, Katayama H, Yokoyama M, Morita K, Akagi R and Sassa S: Highly liver-specific heme oxygenase-1 induction by interleukin-11 prevents carbon tetrachloride-induced hepatotoxicity. Int J Mol Med 18: 537-546, 2006.

38. Lee JW, Bae CJ, Choi YJ, Kim SI, Kwon YS, Lee HJ, Kim SS and Chun W: 3,4,5-trihydroxycinnamic acid inhibits lipopolysaccharide (LPS)-induced inflammation by Nrf2 activation in vitro and improves survival of mice in LPS-induced endotoxemia model in vivo. Mol Cell Biochem 390: 143-153, 2014.

39. BoonlohK,LeeES,KimHM,KwonMH,Kim YM,PannangpetchP, Kongyingyoes B, Kukongviriyapan U, Thawornchinsombut S, Lee EY, et al: Rice bran protein hydrolysates attenuate diabetic nephropathy in diabetic animal model. Eur J Nutr: Dec 21, 2016 (Epub ahead of print). doi: 10.1007/s00394-016-1366-y.

40. Kim SE, Lee EO, Yang JH, Kang JHL, Suh YH and Chong YH: 15 -deoxy- $\Delta^{12,14}$-prostaglandin $\mathrm{J}_{2}$ inhibits human immunodeficiency virus-1 tat-induced monocyte chemoattractant protein-1/CCL2 production by blocking the extracellular signal-regulated kinase-1/2 signaling pathway independently of peroxisome proliferatoractivated receptor- $\gamma$ and heme oxygenase-1 in rat hippocampal slices. J Neurosci Res 90: 1732-1742, 2012.

41. Mouzaoui S, Djerdjouri B, Makhezer N, Kroviarski Y,El-Benna J and Dang PM: Tumor necrosis factor- $\alpha$-induced colitis increases NADPH oxidase 1 expression, oxidative stress, and neutrophil recruitment in the colon: Preventive effect of apocynin. Mediators Inflamm 2014: 312484, 2014

42. Maldonado PD, Molina-Jijón E, Villeda-Hernández J, Galván-Arzate S, Santamaría A and Pedraza-Chaverrí J: $\mathrm{NAD}(\mathrm{P}) \mathrm{H}$ oxidase contributes to neurotoxicity in an excitotoxic/prooxidant model of Huntington's disease in rats: Protective role of apocynin. J Neurosci Res 88: 620-629, 2010.

43. Hamilton CA, Brosnan MJ, Al-Benna S, Berg G and Dominiczak AF: NAD(P)H oxidase inhibition improves endothelial function in rat and human blood vessels. Hypertension 40 755-762, 2002.

44. Park YM, Park MY, Suh Y-L and Park JB: NAD(P)H oxidase inhibitor prevents blood pressure elevation and cardiovascular hypertrophy in aldosterone-infused rats. Biochem Biophys Res Commun 313: 812-817, 2004.

45. Adler S and Huang H: Oxidant stress in kidneys of spontaneously hypertensive rats involves both oxidase overexpression and loss of extracellular superoxide dismutase. Am J Physiol Renal Physiol 287: F907-F913, 2004.

46. Cruz-Álvarez S, Santana-Martínez R, Avila-Chávez E, Barrera-Oviedo D, Hernández-Pando R, Pedraza-Chaverri J and Maldonado PD: Apocynin protects against neurological damage induced by quinolinic acid by an increase in glutathione synthesis and Nrf2 levels. Neuroscience 350: 65-74, 2017.

47. Qiu J, Zhao J, Li J, Liang X, Yang Y, Zhang Z, Zhang X, Fu H, Korantzopoulos P, Tse G, et al: Apocynin attenuates left ventricular remodeling in diabetic rabbits. Oncotarget 8 : 38482-38490, 2017. 